\title{
Association study of sepiapterin reductase gene promoter polymorphisms with schizophrenia in a Han Chinese population
}

This article was published in the following Dove Press journal:

Neuropsychiatric Disease and Treatment

28 October 2015

Number of times this article has been viewed

Jiawu Fu',*
Guoda Ma',*
Hui Mai,*
Xudong Luo'
Jingwen Yin ${ }^{2}$
Qing Chen ${ }^{2}$
Zhixiong Lin ${ }^{2}$
Hua Tao'
You Li'
Lili Cui'
Zheng Li
Juda Lin ${ }^{2}$
Bin Zhao'
Keshen Li'
'Institute of Neurology, Affiliated
Hospital of Guangdong Medical
University, ${ }^{2}$ Department of Psychiatry,
Affiliated Hospital of Guangdong
Medical University, Zhanjiang, People's
Republic of China; ${ }^{3}$ Unit on Synapse
Development and Plasticity, National
Institute of Mental Health, National
Institutes of Health, Bethesda,
MD, USA
*These authors contributed equally
to this work
to this work

\begin{abstract}
Sepiapterin reductase participates in the biosynthesis of tetrahydrobiopterin, which plays very important roles in the pathogenesis of schizophrenia via dysregulation of neurotransmitter systems. Here, two single nucleotide polymorphisms (rs1876487 and rs2421095) in the promoter region of $S P R$ were genotyped in 941 schizophrenic patients and 944 controls in a Han Chinese population using the SNaPshot technique. No significant differences were found in the distribution of alleles or genotypes of the two single nucleotide polymorphisms (SNPs) between schizophrenic patients and controls (all $P>0.05$ ). Likewise, no haplotype was found to be associated with schizophrenia. However, sex-stratified analysis revealed that the frequencies of the A allele of rs1876487 and the A-A (rs2421095-rs1876487) haplotype were all significantly different between schizophrenia and controls in females ( $P=0.040$ and $P=0.033$, respectively), but not in males. Additionally, luciferase reporter gene assays revealed that the $\mathrm{A}-\mathrm{A}$ haplotype had significantly higher $S P R$ transcriptional activity compared with the A-C haplotype in SH-SY5Y cells. Our data indicate that the two SNPs do not influence the risk of schizophrenia when using the total sample, but the A allele of rs1876487 and the A-A haplotype may contribute to protective roles for schizophrenia in females.
\end{abstract}

Keywords: schizophrenia, sepiapterin reductase, polymorphisms, Han Chinese population, transcriptional activity

\section{Introduction}

Schizophrenia is a complex mental disorder associated with a lifetime risk of approximately $1 \%$, characterized by various abnormalities in thought, emotion, and behavior. ${ }^{1}$ In recent years, several mechanisms for the pathophysiology of schizophrenia have been hypothesized, including altered homeostasis of different neurotransmitters, such as dopamine (DA), serotonin (5-HT), and glutamate (Glu), in different cortical and limbic and extrapyramidal pathways. ${ }^{2-4}$ In this regard, abnormalities in enzymatic activities involved in the biosynthesis or release of neurotransmitters may influence the functioning of neurotransmitters in the central nervous system and lead to psychiatric disorders including schizophrenia. .,6 $^{-1}$

Tetrahydrobiopterin $\left(\mathrm{BH}_{4}\right)$, as a critical cofactor, is essentially required for the enzymatic activities of phenylalanine hydroxylase, tyrosine hydroxylase, and tryptophan hydroxylase; the last two enzymes participate in the rate-limiting steps of the biosynthesis of the monoamine neurotransmitter DA and 5-HT, respectively. ${ }^{7}$ Additionally, the increased release of DA, 5-HT, and Glu stimulated by an enhancement of intracellular $\mathrm{BH}_{4}$ has been reported repeatedly, suggesting that $\mathrm{BH}_{4}$ is a regulator of neurotransmitter release. ${ }^{8,9}$ In recent years, a significant plasma deficit
Correspondence: Bin Zhao; Keshen Li Institute of Neurology, Affiliated Hospital of Guangdong Medical University,

Zhanjiang, 52400I, Guangdong,

People's Republic of China

Tel +86 $7592386772 ; 867592386949$

Fax +86759238 6772; 867592386949

Email binzhaoe@।63.com;

likeshenI97I@I26.com 
of total biopterin (a measure of $\mathrm{BH}_{4}$ ) was reported in a large group of schizophrenic patients compared to healthy controls. ${ }^{10,11}$ Convergent animal and human evidence suggested that abnormalities in the biochemical cascades regulated by $\mathrm{BH}_{4}$ possibly altered the neurotransmission of DA, 5-HT, and Glu, consequently contributing to the etiology of schizophrenia.

Sepiapterin reductase (SPR) participates in the final step of the pathway for de novo $\mathrm{BH}_{4}$ biosynthesis. ${ }^{7}$ In a mouse model, $S P R$ deficiency $\left(S P R^{-/-}\right)$led to a shortage of $\mathrm{BH}_{4}$ in the brain, which was reduced to $40.5 \%$ compared to wildtype littermates. ${ }^{12}$ Additionally, $S P R^{-/-}$mice exhibited disrupted homeostasis of DA and 5-HT that was accompanied by increased (three to four times) and sustained locomotor activity. ${ }^{13}$ Furthermore, human patients with SPR deficiency had low $\mathrm{BH}_{4}$ levels in the cerebrospinal fluid. ${ }^{14}$ Based on the above evidence, we speculate that alterations in functional activity or the SPR expression level may represent the missing link that connects the reported abnormalities in $\mathrm{BH}_{4}$ with the pathophysiology of schizophrenia.

$S P R$, the gene coding for SPR, is located on chromosome 2 p13 and is composed of three exons within a $4.7 \mathrm{~kb}$ region. ${ }^{15}$ Karamohamed et al ${ }^{16}$ identified two single nucleotide polymorphisms (SNPs) (rs1876487 and rs2421095) in the promoter region of $S P R$, which may exert functional effects on the basal transcriptional activity and involve the regulation of $S P R$ expression. In an in vitro study, McHugh et $\mathrm{al}^{17}$ showed that the $\mathrm{A}-\mathrm{A}$ and $\mathrm{G}-\mathrm{A}$ haplotypes (rs2421095-rs1876487) all significantly displayed lower expression levels in the basal SPR transcription rate than the A-C haplotype in both COS-7 and RN46A cells. Additionally, McHugh et al verified that individuals with haplotype pairs that consisted of the $\mathrm{A}-\mathrm{A}$ and $\mathrm{G}-\mathrm{A}$ haplotypes would increase the risk of bipolar disorder. ${ }^{17}$ Therefore, we hypothesized that polymorphism(s) in the SPR gene promoter may be responsible for the reduction in enzyme activity and the predisposition to psychiatric diseases, thus constituting a genetic risk factor for schizophrenia.

To our knowledge, a recent genome-wide association studies data analysis revealed that the two SNPs (rs1876487 and rs2421095) in the SPR promoter are not associated with schizophrenia susceptibility in the European population. ${ }^{18}$ However, an associated study between the two SNPs in the $S P R$ gene and schizophrenia in the Han Chinese population has yet to be reported. Hence, we performed a study to explore the relationship between the two aforementioned SNPs of the $S P R$ gene and schizophrenia in a case-control sample of Chinese subjects. Additionally, given the accumulating evidence suggesting that haplotypes have more power to detect associations with the risk of complex diseases compared to single polymorphisms, we undertook an in vitro study to compare the transcriptional activities of $S P R$ with different haplotypes in SH-SY5Y cells.

\section{Materials and methods Subjects}

In this study, 941 unrelated schizophrenic patients (607 males and 334 females; mean age $35.50 \pm 13.81$ years) and 944 healthy controls (598 males and 346 females; mean age $35.62 \pm 12.96$ years) were recruited from the Department of Psychology and the Health Examination Center of the First Affiliated Hospital of Guangdong Medical College, respectively. The healthy controls were well matched to the schizophrenic patients based on sex and age ( $P=0.601$ and 0.848 , respectively). All subjects were Han Chinese in origin. The patients were diagnosed by at least two psychiatrists according to the Diagnostic and Statistical Manual of Mental Disorders IV criteria for schizophrenia on the basis of structured clinical interviews and reviews of their hospital medical records. At the same time, the clinical symptomatology of the patients was assessed using the Positive and Negative Syndrome Scale. None of the subjects had histories of mental retardation, organic brain damage, drug or alcohol abuse, neurological disorders, autoimmune disorders, and metabolic illnesses. All healthy controls were interviewed by a psychiatrist and screened using unstructured clinical interviews. Additionally, they lacked family histories (first-degree relatives) of psychiatric illness, serious somatic illness, and substance abuse diagnosis (excluding nicotine dependence). The study protocol was approved by the Ethics Committee of Guangdong Medical University and written informed consent was obtained from all participants. Finally, the patients' capacity to consent was confirmed by the family member when required.

\section{Genotyping}

Genomic DNA was extracted from ethylene diamine tetraacetic acid-treated peripheral blood using the TIANamp Blood DNA Kit (Tiangen Biotech, Beijing, People's Republic of China). In total, 1,885 individuals were genotyped for two SNPs (rs1876487 and rs2421095) located in the SPR gene using the SNaPshot technique (Thermo Fisher Scientific, Waltham, MA, USA) as described previously. ${ }^{19}$ The polymerase chain reaction primers used for rs 1876487 were 5'-AGGGCTGGAACTGGGAGGAAAT-3' (forward primer) and 5'-TGGTTCCCTTGGGATCTGGTTC-3' (reverse primer), and the primers used for rs2421095 were 5'-CCTCCAAGTTGTTTCTTCCTTAGAGTTG-3' and 5'-TTAGCCCARTTCCCCACAGG-3'. 


\section{Cloning and dual luciferase assays}

Initially, an approximately $1,230 \mathrm{bp}$ region including a 5'-UTR genomic region of SPR (-1204 to $+26 \mathrm{bp}$ ) from an individual A homozygous for rs241095 and rs1876487 was amplified by polymerase chain reaction using Expand High Fidelity DNA polymerase (Roche) and a pair of primers 5'-CGGGGTACCCAGTTTGGGCCACAGAGCGAGACT C-3' and 5'-GGAAGATCTTGGCACCCGAGACCAGGCA GGAGGC- $3^{\prime}$. The resulting haplotype served as the reference haplotype A-A. The genomic fragments amplified with the Kpn1 and BglII sites were cloned into a pGL4 Basic Vector (Promega Corporation, Fitchburg, WI, USA). The C polymorphism of rs 1876487 was then sequentially altered using a QuikChange II site-directed mutagenesis kit (Stratagene, Santa Clara, CA, USA) according to the manufacturer's instructions. All of the constructs were confirmed by sequence analysis. The reporter constructs were introduced by Effectene-mediated transfection (Qiagen NV, Venlo, the Netherlands) into the SH-SY5Y neuroblastoma cell lines. Luciferase activity was measured according to our previous description. ${ }^{20}$ Each assay was conducted in triplicate in two independent experiments.

For the functional prediction of the candidate SNP, we used Transcription Element Search System software (http:// www.cbil.upenn.edu/cgi-bin/tess/tess) to analyze potential protein binding sequences containing SNP rs1876487. In order to identify possible changes to transcription factor binding site (TFBS) due to the presence of the rs 1876487 polymorphism, we performed the TFBS in two different situations: a TFBS search when the sequence possessed the ancestral allele $(C)$ and a TFBS search in a sequence in which the polymorphism (A) was present.

\section{Statistical analyses}

The demographic and clinical data between the schizophrenic patients and the controls were compared using Student's $t$-tests or Pearson's chi-squared tests as appropriate. Hardy-Weinberg equilibrium and statistical analyses to compare allelic and genotypic distributions were performed using Pearson's chi-squared tests and Fisher's exact two-tailed tests when appropriate. All analyses were performed using SPSS 17.0 software for Windows. The criterion for significance was set at $P<0.05$ for all of the tests and $P$-values were two-sided.

Additionally, the linkage disequilibrium status of the two SNPs was determined using the Haploview 4.2 program. Only those haplotypes with frequencies greater than $1 \%$ were examined. For the luciferase reporter gene assay data analysis, statistical comparisons between two groups were assessed using Student's $t$-test.

\section{Results \\ Association study of SNPs (rs I 876487 and rs2421095) and schizophrenia}

The distribution of genotypes and alleles of the two SNPs (rs1876487 and rs2421095) reached the Hardy-Weinberg equilibrium in both schizophrenic patients $(P=0.47$ and 0.91 , respectively) and controls ( $P=0.88$ and 0.91 , respectively). Allele and genotype frequencies for the $S P R$ gene are shown in Table 1. In terms of the rs 1876487 polymorphism, the

Table I Genotype and allele frequency of the SPR gene in schizophrenic patients and controls

\begin{tabular}{|c|c|c|c|c|}
\hline dbSNP ID & $\begin{array}{l}\text { Schizophrenic patients, } \\
\mathbf{N}=94 \text { I, n (\%) }\end{array}$ & $\begin{array}{l}\text { Control patients, } \\
\mathrm{N}=944, \mathrm{n}(\%)\end{array}$ & $P$-value & OR $(95 \% \mathrm{CI})$ \\
\hline \multicolumn{5}{|l|}{ rsI876487 } \\
\hline \multicolumn{5}{|l|}{ Genotype } \\
\hline $\mathrm{CC}$ & $856(91.0)$ & $845(89.5)$ & & 1.000 (reference) \\
\hline $\mathrm{CA}$ & $84(8.9)$ & $96(10.2)$ & & \\
\hline AA & $\mathrm{I}(0.1)$ & $3(0.3)$ & & \\
\hline $\mathrm{CA}+\mathrm{AA}$ & $85(9.0)$ & $99(10.5)$ & $0.287^{\mathrm{a}}$ & $0.848(0.625-1.150)$ \\
\hline \multicolumn{5}{|l|}{ Allele } \\
\hline $\mathrm{C}$ & I,796 (95.4) & I,786 (94.6) & & 1.000 (reference) \\
\hline$A$ & $86(4.6)$ & $102(5.4)$ & 0.240 & $0.838(0.625-1.125)$ \\
\hline \multicolumn{5}{|l|}{ rs2421095 } \\
\hline \multicolumn{5}{|l|}{ Genotype } \\
\hline AA & $934(99.3)$ & $937(99.3)$ & & 1.000 (reference) \\
\hline AG & $7(0.7)$ & $7(0.7)$ & $0.995^{\mathrm{b}}$ & $\mathrm{I} .003(0.35 \mathrm{I}-2.87 \mathrm{I})$ \\
\hline GG & $0(0.0)$ & $0(0.0)$ & & \\
\hline \multicolumn{5}{|l|}{ Allele } \\
\hline A & I,875 (99.6) & I,88I (99.6) & & 1.000 (reference) \\
\hline G & $7(0.4)$ & $7(0.4)$ & 0.995 & $\mathrm{I} .003(0.35 \mathrm{I}-2.866)$ \\
\hline
\end{tabular}

Notes: a ${ }^{\mathrm{C}}$ alculations were performed using $\mathrm{CA}+\mathrm{AA}$ vs $\mathrm{CC}$. ${ }^{\mathrm{b}} \mathrm{Calculations}$ were performed using $\mathrm{AG}$ vs $\mathrm{AA}$. Abbreviations: dbSNP, Single Nucleotide Polymorphism Database; OR, odds ratio; $\mathrm{Cl}$, confidence interval. 
respective frequencies of $\mathrm{CC}, \mathrm{CA}$, and $\mathrm{AA}$ genotypes were $91.0 \%, 8.9 \%$, and $0.1 \%$ in the schizophrenic patients, and $89.5 \%, 10.2 \%$, and $0.3 \%$ in the control group, respectively. Due to the low frequency of the AA genotype, the AA genotypes were grouped with the CA genotypes and were referenced as A allele carriers. For rs2421095, none of the individuals had the GG genotype in our study population, and the frequencies for the $\mathrm{AA}$ and $\mathrm{AG}$ genotypes in the schizophrenic patients and the controls were almost identical ( $99.3 \%$ and $0.7 \%$, respectively). There were no significant differences in the distribution of the two aforementioned SNPs between our schizophrenic patients and controls (all $P>0.05$ ). Additionally, in the sex-stratified analysis, the genotype and allele distributions of rs 1876487 were significantly different between schizophrenia and controls in females ( $P=0.045$ and 0.040 , respectively), but not in males (Table 2).

For further analysis, our study also focused on the description of relevant clinical characteristics of 543 schizophrenic patients distributed by genotype. None of the CC genotype and A allele carriers of rs 1876487 showed significant associations with clinical characteristics, such as age at onset, duration of illness, Positive and Negative Syndrome Scale score, family psychotic history, and other factors (Table 3 ).

\section{Linkage disequilibrium analysis and haplotype analysis}

We also performed a haplotype analysis based on the two SNPs rs2421095 and rs1876487, and a weak linkage disequilibrium was observed $\left(\mathrm{D}^{\prime}=0.607, r^{2}=0.026\right)$. Two out of four possible haplotypes revealed at least $1 \%$ frequencies in either the schizophrenic patients or the controls. Analysis of the haplotype frequencies demonstrated no significant difference between the schizophrenic patients and controls. Furthermore, in the female subgroup, the A-A (rs2421095-rs1876487) haplotype was significantly different between the schizophrenic patients and the controls $(P=0.033$, odds ratio $[\mathrm{OR}]=0.575$, $95 \%$ confidence interval $[\mathrm{CI}]=0.344-0.961)$ (Table 4$)$.

\section{Functional analysis of different SPR promoter haplotypes}

As shown in Figure 1, the promoter activity driven by the A-A haplotype was 1.2-fold higher than the A-C haplotype, and the difference was significant $(P<0.05)$. Our data suggested that the A allele of rs 1876487 might improve the $S P R$ promoter activity.

To clarify the underlying molecular mechanism of the influence of SNPs on SPR promoter activity, the putative

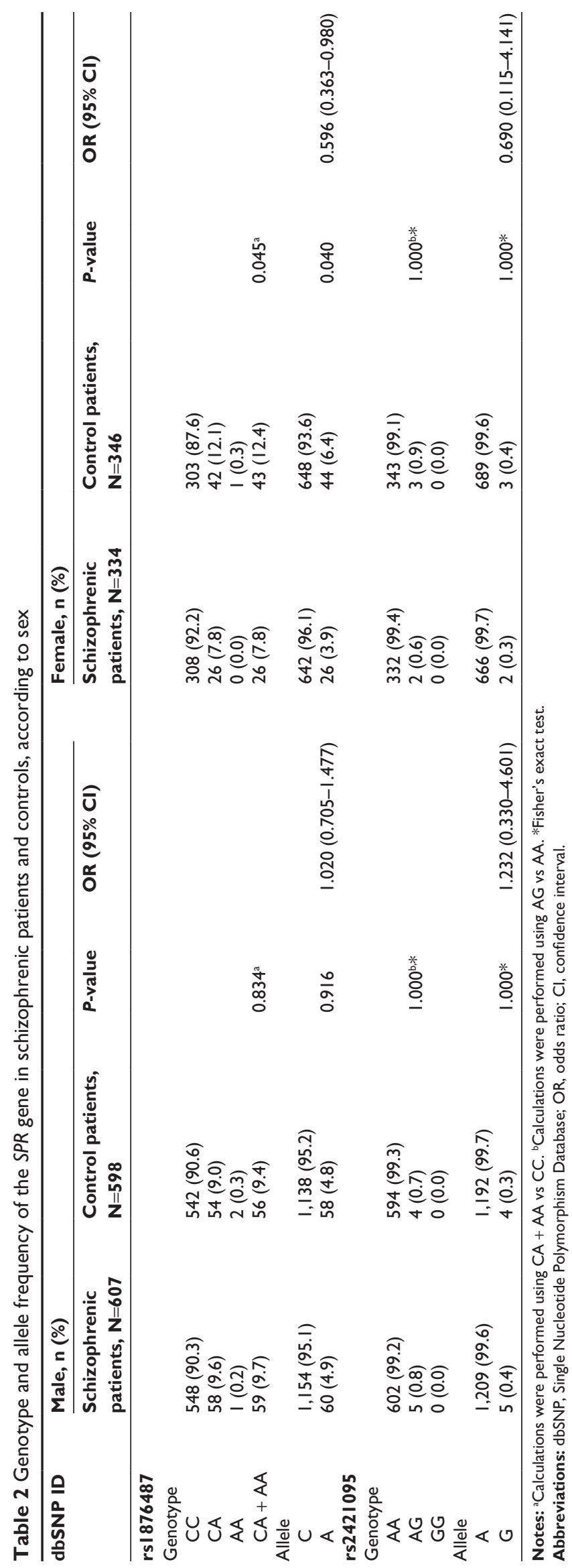


Table 3 Clinical characteristics of schizophrenic patients and distribution by genotypes of rs 1876487

\begin{tabular}{|c|c|c|c|c|}
\hline \multirow[t]{2}{*}{ Parameters } & \multicolumn{4}{|l|}{ rs 1876487} \\
\hline & CC $(n=493)$ & $\mathrm{CA}+\mathrm{AA}(n=50)$ & $t / \chi^{2}$ & $P$-value \\
\hline Age at onset (years) & $25.11 \pm 9.82$ & $26.26 \pm 11.63$ & 0.775 & 0.439 \\
\hline Duration of illness (years) & $11.65 \pm 9.94$ & $11.10 \pm 10.56$ & 0.368 & 0.713 \\
\hline Education (years) & $9.61 \pm 2.84$ & $9.54 \pm 3.17$ & 0.175 & $0.86 I$ \\
\hline PANSS total score & $76.61 \pm 16.37$ & $81.90 \pm 19.60$ & 1.964 & $0.05 I$ \\
\hline P subscore & $22.32 \pm 7.17$ & $23.24 \pm 7.75$ & 0.856 & 0.392 \\
\hline $\mathrm{N}$ subscore & $18.00 \pm 7.45$ & $20.10 \pm 8.11$ & 1.884 & 0.060 \\
\hline G subscore & $36.29 \pm 8.50$ & $38.56 \pm 9.71$ & 1.772 & 0.077 \\
\hline $\mathrm{P}$ subscore- $\mathrm{N}$ subscore $>0$ & $339(68.8 \%)$ & $34(68.0 \%)$ & 0.012 & 0.912 \\
\hline Family psychotic history & $78(15.8 \%)$ & $5(10.0 \%)$ & 1.188 & 0.276 \\
\hline \multicolumn{5}{|l|}{ Age at onset (years) } \\
\hline$<18$ & 94 (19.1\%) & $13(26.0 \%)$ & 1.379 & 0.240 \\
\hline$\geq 18$ & $399(80.9 \%)$ & 37 (74.0\%) & NA & NA \\
\hline
\end{tabular}

Note: Values are mean \pm SD.

Abbreviations: G, General Psychopathology scale; N, Negative scale; NA, not applicable; P, Positive scale; PANSS, Positive and Negative Syndrome Scale; SD, standard deviation.

TFBS were performed using Transcription Element Search System software. The analysis (data not shown) predicted that the $\mathrm{C}$ allele of rs 1876487 would generate an SP1-binding site, but the A allele would not.

\section{Discussion}

Given the critical role of SPR in neurotransmitter production, we sought to test the hypothesis that SPR would be involved in the pathogenesis of schizophrenia. In this study, the genetic analysis of $S P R$ polymorphisms between the schizophrenic patients and the controls indicated that there were no significant differences in terms of the distribution of the rs1876487 and rs2421095 genotypes and alleles. Additionally, we have demonstrated that the GG genotype (rs2421095) was not detected in either the patients or the controls in our study. Furthermore, the A allele frequency of rs 1876487 and the $G$ allele frequency of rs 2421095 were lower in our Chinese population (5.4\% and $0.4 \%)$ than the corresponding frequencies in the USA $(21.9 \%$ and $4.7 \%)^{21}$ and New Zealand (30.0\% and $\left.12.0 \%\right),{ }^{17}$ but were similar to those in the Chinese Beijing population of the International HapMap Project (http://hapmap.ncbi.nlm.nih. gov/). The results suggest that there is an ethnicity-related variation in the distribution of the two aforementioned SNPs in the SPR gene. Our findings suggest that the two polymorphisms may not contribute to schizophrenia susceptibility in the Han Chinese population. However, we did not clarify the relationship between other SNPs in the $S P R$ gene and schizophrenia. Based on these, the identification of other polymorphisms in the SPR gene associated with schizophrenia and replication in other ethnic populations requires further study.

Interestingly, the stratified analysis by sex revealed that the genotype and allele frequencies of rs 1876487 between schizophrenic patients and controls in females showed significant differences ( $P=0.045$ and 0.040 , respectively), but

Table 4 Haplotype analysis of two SNPs in the SPR gene in schizophrenic patients and controls

\begin{tabular}{|c|c|c|c|c|}
\hline \multirow[t]{2}{*}{ rs242 I 095-rs I 876487} & \multicolumn{2}{|l|}{ Frequencies, n (\%) } & \multirow[t]{2}{*}{$P$-value } & \multirow[t]{2}{*}{ OR $(95 \% \mathrm{Cl})$} \\
\hline & Schizophrenic patients & Control patients & & \\
\hline \multicolumn{5}{|l|}{ Total } \\
\hline$A-C$ & I,792.9 (95.3) & I,783.9 (94.5) & & 1.000 (reference) \\
\hline$A-A$ & $82.1(4.4)$ & $97.1(5.1)$ & 0.260 & $0.84 \mid(0.622-1.137)$ \\
\hline \multicolumn{5}{|l|}{ Male } \\
\hline$A-C$ & I,I $50.9(94.8)$ & I,I $36.9(95.1)$ & & 1.000 (reference) \\
\hline$A-A$ & $58.1(4.8)$ & $55.1(4.6)$ & 0.832 & $1.042(0.7 \mid 4-1.520)$ \\
\hline \multicolumn{5}{|l|}{ Female } \\
\hline $\mathrm{A}-\mathrm{C}$ & $642.0(96.1)$ & $646.0(93.6)$ & & 1.000 (reference) \\
\hline$A-A$ & $24.0(3.6)$ & $42.0(6.1)$ & 0.033 & $0.575(0.344-0.961)$ \\
\hline
\end{tabular}

Note: Haplotype frequency $<0.0 \mathrm{I}$ in both schizophrenic patients and controls has been dropped.

Abbreviations: SNP, single nucleotide polymorphism; OR, odds ratio; $\mathrm{Cl}$, confidence interval. 


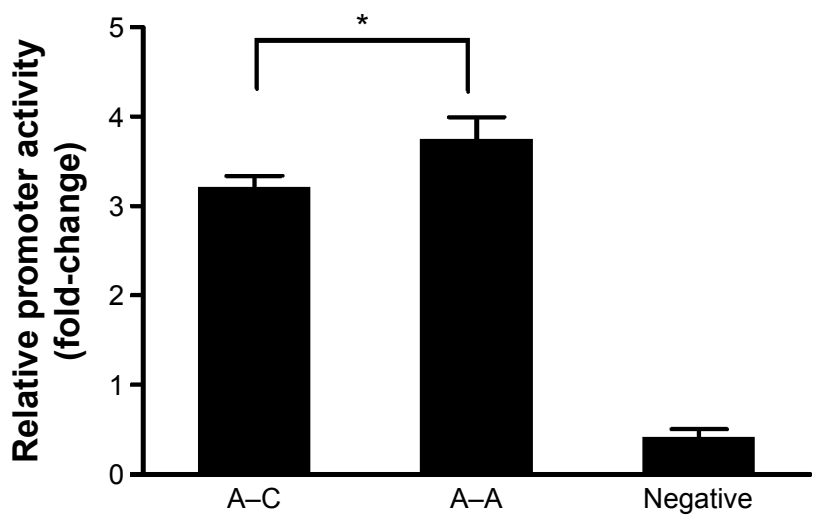

Figure I Reporter gene analysis of sepiapterin reductase haplotypes.

Notes: The expression levels of two promoter constructs containing the A-C or A-A haplotypes (rs2421095-rs1876487) were compared in SH-SY5Y cells. The expression levels of each haplotype with standard deviations are shown. The negative control was the empty $p G L 4$-basic vector. $* P<0.05$.

not in males. These results are likely to suggest a sex specific association for rs 1876487 of the SPR gene with schizophrenia. Our finding is not surprising because many studies have reported that there were sex differences in the pathogenesis of schizophrenia. Grigoriadis and Seeman ${ }^{22}$ suggested that estrogen exerts a protective effect against schizophrenia. Additionally, estrogen regulates the dopaminergic and serotonergic activities in different brain regions of patients with schizophrenia. ${ }^{23}$ In this study, the frequency of the A allele of rs1876487 in the schizophrenic patients was lower compared to the controls in the female subgroup (3.9\% vs $6.4 \%$, OR $=0.596,95 \% \mathrm{CI}=0.363-0.980)$, suggesting that the A allele of rs1876487 may have a protective role in female schizophrenia. Additionally, we predicted that the $\mathrm{C}$ allele of rs1876487 generated a SP1-binding site, but the A allele would not. It has been reported that SP1 could activate the expression of some genes and downregulate that of other genes. ${ }^{24}$ Additionally, SP1 is involved in the regulation of several genes related to schizophrenia, such as DA receptors and mitochondrial complex I genes. ${ }^{25}$ However, the potential relationship between SP1 and SPR expression is not yet understood. Furthermore, a study by Sumi and Ignarro verified that estrogen-related receptor $\alpha$ increases the SP1 expression in COS-7 cells. ${ }^{26}$ Taken together, it can be speculated that estrogen stimulates SP1 expression through estrogen-related receptor $\alpha$, and then regulates SPR gene transcription in females. However, further investigation is required to clarify the interaction between estrogen and the SPR gene in the central nervous system. Analysis of haplotype-based association in our genetic study revealed that no significant differences existed in the distribution of the
A-C and A-A haplotypes between the schizophrenic patients and the controls. Intriguingly, in our female subjects, the frequency of the A-A haplotype was higher in the controls $(6.1 \%$ vs $3.6 \%$ ), indicating that this haplotype may constitute a protective factor for schizophrenia $(P=0.033$, OR $=0.575,95 \%$ $\mathrm{CI}=0.344-0.961)$. This result is consistent with our functional analysis that the $\mathrm{A}-\mathrm{A}$ haplotype significantly induced higher $S P R$ transcriptional activity compared with $\mathrm{A}-\mathrm{C}$ haplotype in SH-SY5Y cells, which would lead to increased SPR expression and upstream effects on $\mathrm{BH}_{4}$-mediated neurotransmitter production. However, in a previous study, McHugh et al $^{17}$ reported that the A-A haplotype displayed significantly lower expression levels in the basal transcription rate of the $S P R$ gene than the A-C haplotype in both COS-7 and RN46A cells. Several reasons may partially account for such conflicting results. First, different cell types or tissues may have diverse transfection activities. Second, the two SNPs in different ethnic populations share different $\mathrm{D}^{\prime}$ and $r^{2}$ values, and may thus have different levels of linkage disequilibrium with other functional SNPs, which alter the promoter activity of the $S P R$ gene. However, more studies are still needed to elucidate the function of the two-SNP SPR promoter haplotypes in various cell lines.

\section{Conclusion}

The results from our study might provide evidence that the two SNPs (rs1876487 and rs2421095) in the SPR promoter are not associated with schizophrenia susceptibility in the Han Chinese population. However, the A allele of rs 1876487 and the A-A haplotype may contribute to protective roles in female schizophrenia. Thus, to confirm our finding, replication in a large sample size, especially for females and other ethnic populations, is required. Furthermore, the identification of other polymorphisms in the SPR gene associated with schizophrenia would warrant further investigation.

\section{Acknowledgments}

This work was supported by funding from the US-China Biomedical Collaborative Research Program (grant number 81261120404), the National Nature Science Foundation of China (grant numbers 31171219, 81271213, 81070878, and 81271214, and 81471294), and the Science and Technology Innovation Fund of Guangdong Medical University (numbers STIF 201101 and M2014047).

\section{Disclosure}

The authors report no conflicts of interest in this work. 


\section{References}

1. van Os J, Kapur S. Schizophrenia. Lancet. 2009;374(9690):635-645.

2. Puig MV, Gulledge AT. Serotonin and prefrontal cortex function: neurons, networks, and circuits. Mol Neurobiol. 2011;44(3):449-464.

3. Howes OD, Kapur S. The dopamine hypothesis of schizophrenia: version III-the final common pathway. Schizophr Bull. 2009;35(3):549-562.

4. Tsai G, Coyle JT. Glutamatergic mechanisms in schizophrenia. Annu Rev Pharmacol Toxicol. 2002;42:165-179.

5. Li D, He L. Meta-analysis shows association between the tryptophan hydroxylase (TPH) gene and schizophrenia. Hum Genet. 2006; 120(1):22-30.

6. Srivastava V, Deshpande SN, Thelma BK. Dopaminergic pathway gene polymorphisms and genetic susceptibility to schizophrenia among north Indians. Neuropsychobiology. 2010;61(2):64-70.

7. Werner ER, Blau N, Thony B. Tetrahydrobiopterin: biochemistry and pathophysiology. Biochem J. 2011;438(3):397-414.

8. Mataga N, Imamura K, Watanabe Y. 6R-tetrahydrobiopterin perfusion enhances dopamine, serotonin, and glutamate outputs in dialysate from rat striatum and frontal cortex. Brain Res. 1991;551(1-2):64-71.

9. Abreu-Gonzalez P, Gonzalez-Hernandez T, Afonso-Oramas D, Cruz-Muros I, Barroso-Chinea P, Gonzalez MC. Tetrahydrobiopterin stimulates L-DOPA release from striatal tissue. Eur J Pharmacol. 2006;541(1-2):33-37.

10. Richardson MA, Read LL, Taylor Clelland CL, et al. Evidence for a tetrahydrobiopterin deficit in schizophrenia. Neuropsychobiology. 2005; 52(4):190-201.

11. Richardson MA, Read LL, Reilly MA, Clelland JD, Clelland CL. Analysis of plasma biopterin levels in psychiatric disorders suggests a common BH4 deficit in schizophrenia and schizoaffective disorder. Neurochem Res. 2007;32(1):107-113.

12. Yang S, Lee YJ, Kim JM, et al. A murine model for human sepiapterinreductase deficiency. Am J Hum Genet. 2006;78(4):575-587.

13. Choi YK, Tarazi FI. Alterations in dopamine and glutamate neurotransmission in tetrahydrobiopterin deficient spr-/-mice: relevance to schizophrenia. BMB Reports. 2010;43(9):593-598.

14. Bonafe L, Thony B, Penzien JM, Czarnecki B, Blau N. Mutations in the sepiapterin reductase gene cause a novel tetrahydrobiopterin-dependent monoamine-neurotransmitter deficiency without hyperphenylalaninemia. Am J Hum Genet. 2001;69(2):269-277.
15. Thony B, Auerbach G, Blau N. Tetrahydrobiopterin biosynthesis, regeneration and functions. Biochem J. 2000;347 Pt 1:1-16.

16. Karamohamed S, DeStefano AL, Wilk JB, et al. A haplotype at the PARK3 locus influences onset age for Parkinson's disease: the GenePD study. Neurology. 2003;61(11):1557-1561.

17. McHugh PC, Joyce PR, Kennedy MA. Polymorphisms of sepiapterin reductase gene alter promoter activity and may influence risk of bipolar disorder. Pharmacogenet Genomics. 2009;19(5):330-337.

18. Ripke S, O'Dushlaine C, Chambert K, et al. Genome-wide association analysis identifies 13 new risk loci for schizophrenia. Nat Genet. 2013;45(10):1150-1159.

19. Li Y, Liao F, Yin XJ, et al. An association study on ADAM10 promoter polymorphisms and atherosclerotic cerebral infarction in a Chinese population. CNS Neurosci Ther. 2013;19(10):785-794.

20. Ma G, Yu L, Wang Q, Liu W, Cui Y, Kwang J. Sf-PHB2, a new transcription factor, drives WSSV Ie1 gene expression via a 12-bp DNA element. Virol J. 2012;9:206.

21. Tobin JE, Cui J, Wilk JB, et al. Sepiapterin reductase expression is increased in Parkinson's disease brain tissue. Brain Res. 2007;1139: $42-47$.

22. Grigoriadis S, Seeman MV. The role of estrogen in schizophrenia: implications for schizophrenia practice guidelines for women. Can $J$ Psychiatry. 2002;47(5):437-442.

23. Cyr M, Calon F, Morissette M, Di Paolo T. Estrogenic modulation of brain activity: implications for schizophrenia and Parkinson's disease. J Psychiatry Neurosci. 2002;27(1):12-27.

24. Feng D, Kan YW. The binding of the ubiquitous transcription factor $\mathrm{Sp} 1$ at the locus control region represses the expression of beta-like globin genes. Proc Natl Acad Sci U S A. 2005;102(28):9896-9900.

25. Ben-Shachar D, Karry R. Sp1 expression is disrupted in schizophrenia; a possible mechanism for the abnormal expression of mitochondrial complex I genes, NDUFV1 and NDUFV2. PLoS One. 2007;2(9):e817.

26. Sumi D, Ignarro LJ. Sp1 transcription factor expression is regulated by estrogen-related receptor alpha1. Biochem Biophys Res Commun. 2005;328(1):165-172.
Neuropsychiatric Disease and Treatment

\section{Publish your work in this journal}

Neuropsychiatric Disease and Treatment is an international, peerreviewed journal of clinical therapeutics and pharmacology focusing on concise rapid reporting of clinical or pre-clinical studies on a range of neuropsychiatric and neurological disorders. This journal is indexed on PubMed Central, the 'PsycINFO' database and CAS,

\section{Dovepress}

and is the official journal of The International Neuropsychiatric Association (INA). The manuscript management system is completely online and includes a very quick and fair peer-review system, which is all easy to use. Visit http://www.dovepress.com/testimonials.php to read real quotes from published authors. 